IMAGING

\section{Conformational SNARE}

Proc. Natl. Acad. Sci. USA, published online

12 August 2013; doi:10.1073/pnas.1306699110
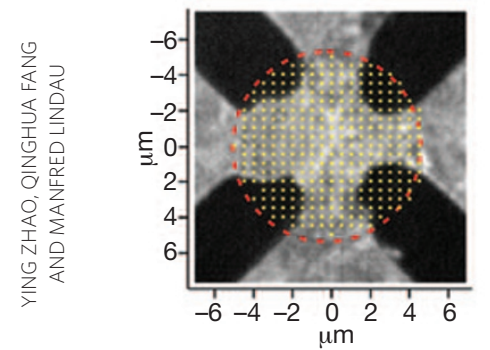

Compounds that mediate signaling across cellular synapses are released by exocytosis when the secretory vesicles that contain them form a fusion pore with the plasma membrane. Three proteins, including synaptosomal-associated protein 25 (SNAP25), make up the SNARE complex, which drives the formation of the fusion pore during exocytosis. The fluorescence resonance energy transfer (FRET)-based SNARE complex reporter (SCORE), a fusion protein containing SNAP25, CFP and Venus, was previously developed to assess whether conformational changes in SNARE are linked to fusion pore formation, but the time resolution afforded by the application of this tool exceeded the timing of the fusion event. Zhao et al. now develop event correlation microscopy (ECOM) that improves time resolution to $\sim 1 \mathrm{~ms}$. In the authors' system, time and location of single fusion events in cells expressing SCORE were monitored in tandem. They recorded images from total internal reflection FRET microscopy and overlaid the images with maps of catecholamine release, which marks fusion events, derived from data collected via a four-electrode electrochemical detector. The authors collected data from over 900 fusion events, revealing a consistent $~ 90$-ms delay between FRET changes in SCORE and the amperometric spike associated with catecholamine release. Collectively, the data are consistent with a rapid conformational change in the SNARE complex preceding the opening of the fusion pore by milliseconds.

$A D$

\section{NEURODEGENERATION}

\section{Tickled PINK1}

\section{Cell 154, 737-747 (2013)}

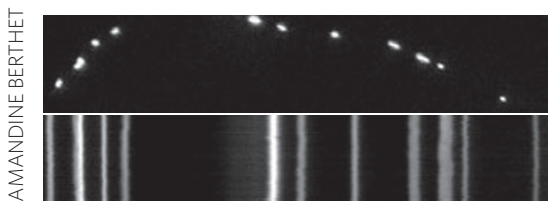

Parkinson's disease (PD) is a progressive neurological disorder in which the substantia nigra region of the brain is depleted in dopaminergic neurons. Mitochondrial defects have long been associated with PD. For example, mutations in PTEN-induced kinase 1 (PINK1), which is involved in mitochondrial maintenance in neurons, have been linked to an earlyonset form of $\mathrm{PD}$, prompting suggestions that enhancers of PINK1 activity could be neuroprotective. Hertz et al. now identify a nucleotide triphosphate 'neo-substrate' that elevates PINK1 activity in neurons and provide cellular data in support of this

\section{PROTEIN DYNAMICS}

\section{Shake it up, baby}

therapeutic model. Examination of PINK1 sequences, particularly of the G309D mutant associated with PD, led the authors to hypothesize that PINK1 enzymes may be able to accommodate larger ATP analogs as substrates. A screen of a small panel of N6-alkyl-ATP analogs revealed that PINK1 and its G309D mutant use the neo-substrate kinetin triphosphate (KTP, N6-furfuryl ATP) more efficiently than ATP itself. Cells treated with kinetin, the nucleoside precursor of KTP, readily accumulate KTP at amounts that restore nearly wild-type activity of PINK1. Finally, the authors showed that neurons treated with kinetin display several molecular phenotypes consistent with PINK1 activation. In addition to validating a potential therapeutic avenue for early-onset $\mathrm{PD}$, the study points toward the possibility that neo-substrate activation could be a general approach for modulating disease-related kinase activity.

\section{DRUG DISCOVERY}

\section{Look within}

Nat. Med., published online 4 Aug 2013; doi:10.1038/nm.3262

\section{Killing Mycobacterium tuberculosis $(M t b)$} inside of host cells is critical for effective treatment of tuberculosis. To find new potential drugs that are capable of doing this, Pethe et al. used an automated microscopy platform to screen a library of 121,156 compounds for those that could interfere with replication of $M t b$ within macrophages. Two compounds with an imidazopyridine amide scaffold were isolated, and they had low micromolar potencies against a reference $M t b$ strain as well as against multidrug-resistant (MDR) clinical isolates. Lead optimization led the authors to Q203, a compound with an MIC50 of $0.28 \mathrm{nM}$ within macrophages that is capable of killing MDR and also extensively drug-resistant (XDR) strains. Q203 was not toxic to macrophages and had other properties that make it an attractive drug lead, including metabolic stability and an absence of drugdrug interactions, and there was a low rate of spontaneous mutation with the potential to lead to resistance in treated $M t b$ cells. Q203 was effective in mouse models of acute and established tuberculosis. Sequencing the genomes of Q203-resistant $M t b$ mutants and an assay monitoring ATP synthesis suggested that the QcrB subunit of the cytochrome $b c_{1}$ complex is the direct efficacy target. These studies demonstrate that Q203 has desirable features for clinical treatment of MDR and XDR tuberculosis. 